\title{
Anabases
}

ANABASES Traditions et réceptions de l'Antiquité

$13 \mid 2011$

Varia

\section{Le monde du Satyricon et la maison de Pline le Jeune}

Stéphane Ratti

\section{OpenEdition}

1 Journals

Édition électronique

URL : http://journals.openedition.org/anabases/1753

DOI : 10.4000/anabases. 1753

ISSN : 2256-9421

Éditeur

E.R.A.S.M.E.

Édition imprimée

Date de publication : 1 mars 2011

Pagination : 79-94

ISSN : 1774-4296

\section{Référence électronique}

Stéphane Ratti, «Le monde du Satyricon et la maison de Pline le Jeune », Anabases [En ligne], 13 |

2011, mis en ligne le 01 mars 2014, consulté le 21 octobre 2019. URL : http://

journals.openedition.org/anabases/1753 ; DOI : 10.4000/anabases.1753

Ce document a été généré automatiquement le 21 octobre 2019

(c) Anabases 


\title{
Le monde du Satyricon et la maison de Pline le Jeune
}

\author{
Stéphane Ratti
}

Le Satyricon s'ouvre pour nous sur une violente et fort savoureuse dénonciation de la baisse générale du niveau des élèves que le système éducatif du temps - à vrai dire les écoles de rhétorique, mais c'est à l'époque la même chose -, et leurs maîtres demisavants, les umbratici doctores $(2,4)$, a profondément décervelés : adulescentulos existimo in scholis stultissimos fieri se lamente Encolpe; "je crains que les jeunes à l'école ne deviennent parfaitement idiots » (sat. 1,3). Le motif est de tous les temps et de tous les lieux et n'a pas même paru incongru à un Victor Hugo dénonçant, dans Les Misérables, l'abrutissement scolaire par la bouche du fort sympathique " philosophe » membre du cercle des « Amis de l'Arc », le dénommé Combeferre :

«Il déclarait que l'avenir est dans la main du maître d'école, et se préoccupait des questions d'éducation. Il voulait que la société travaillât sans relâche à l'élévation du niveau intellectuel et moral, au monnayage de la science, à la mise en circulation des idées, à la croissance de l'esprit dans la jeunesse, et il craignait que la pauvreté actuelle des méthodes, la misère du point de vue littéraire borné à deux ou trois siècles classiques, le dogmatisme tyrannique des pédants officiels, les préjugés scolastiques et les routines ne finisent par faire de nos collèges des huîtrières artificielles ${ }^{1}$.»

2 Le lecteur du Satyricon dans la «Collection des Universités de France » ne se voit pas sans surprise renvoyé par les premières notes de l'éditeur censées éclairer la diatribe d'Encolpe à des auteurs tous postérieurs à l'époque néronienne ${ }^{2}$. A. Ernout, était, on le sait, un partisan de la thèse traditionnelle qui voit en l'auteur du Satyricon le Pétrone mentionné par Tacite (ann. 16, 17-19), soit le « personnage consulaire, contemporain et familier de Néron que la jalousie de Tigellin fit mettre à mort ${ }^{3}$ » en l'an 65 de notre ère. On ne peut qu'être frappé par l'aveugle clairvoyance du célèbre latiniste. Je m'explique : il faut en effet rendre tout d'abord hommage à sa sagacité car tous les rapprochements qu'il propose entre sat. 1-4 et Quintilien (inst. 12, 10, 16 sqq.) ${ }^{4}$ ou Tacite (dial. 28-29) ${ }^{5}$ sont parfaitement probants, sans parler de l'évocation fugace des noms de Martial et de Pline le Jeune ${ }^{6}$, intuition exprimée au passage, fondamentalement juste mais inexplicablement inaboutie puisqu'elle ne débouche sur aucune conclusion. Il faut 
surtout s'étonner de son aveuglement: comment persister dans une datation néronienne du roman alors que la très riche intertextualité du débat sur la décadence de l'enseignement conduit de manière irréfutable au début du $\mathrm{II}^{\mathrm{e}}$ siècle ? Faut-il penser que le poids de la tradition, l'opinio communis et l'autorité académique de quelques maîtres, ont fermé les yeux non au philologue, qui avait vu juste, mais à l'universitaire qui n'avait su tirer toutes les conséquences de ses observations? La leçon, du point de vue épistémologique, me paraît d'une grande force. Mais Pascal, dans les Pensées, l'a dit mieux que je ne saurais le faire : «Ceux qui sont accoutumés à juger par le sentiment ne comprennent rien aux choses du raisonnement, car ils veulent d'abord pénétrer d'une vue et ne sont point accoutumés à chercher les principes ${ }^{7}$. "

Les recherches menées depuis maintenant plusieurs années par René Martin sur la datation du Satyricon ont bouleversé les données traditionnelles du problème et permis des avancées considérables ${ }^{8}$. Il me paraît notamment - je les ai moi-même patiemment vérifiées point par point - que les observations qu'il propose, à la suite de quelques autres, sur les loci similes rencontrés dans le Satyricon d'une part, Silius Italicus, Martial, Tacite (Dialogue des orateurs) et Pline le Jeune d'autre part sont irréfutables ${ }^{9}$. Je ne peux ici les citer tous et je me contenterai de rappeler que le nom même de Trimalchion a été inspiré à l'auteur du Satyricon par la satire 3,82 de Martial où apparaît un débauché du nom de Malchio $^{10}$, mais sans doute trois fois moins dépravé que l'amphitryon de la cena, le nom de ce dernier ayant été fabriqué sur le modèle du Trinummus plautinien, le procédé même qui servira encore à Molière pour baptiser son Trissotin. Tout aussi significatif est le fait que le parfumeur Cosmus cité par Martial dans cette même pièce (et Cosmianis ipse fusus ampullis / non erubescit murice aureo nobis / diuidere moechae pauperis capillare: vers 26-28; "Parfumé des essences que renferment les bocaux de Cosmus, il ne répugne pas à nous distribuer, dans un murex doré, la pommade qui sert aux cheveux d'une infâme courtisane») ait sans aucun doute possible inspiré un passage perdu du Satyricon dont Niccolo Perotti, au $\mathrm{Xv}^{\mathrm{e}}$ siècle, nous a sauvegardé les débris : Cosmus etiam excellens unguentarius fuit, a quo unguenta dicta sunt Cosmiana [...]. Petronius: "Affer nobis, inquit, alabastrum Cosmiani ${ }^{11}$ "; "Cosmus était un grand parfumeur qui a donné son nom aux parfums Cosmaniens [...]. Pétrone dit: "Apportenous une fiole de Cosmanien". » Or Cosmus est parfaitement inconnu avant Martial ${ }^{12}$ et la référence pétronienne au satiriste fournit assurément désormais un point fixe pour la datation du roman après 102 , date de la mort du poète ${ }^{13}$.

4 Je me permettrai d'ajouter à la déjà longue liste des rapprochements incontestables entre Martial et Pétrone établie par René Martin deux points supplémentaires. Le fragment 8 de Pétrone, transmis par Fulgence ${ }^{14}$, nous apprend qu'Ascylte et Encolpe se font servir à boire par Quartilla, au cours de l'épisode licencieux bien connu ${ }^{15}$, dans des vases myrrhins, cette vaisselle précieuse élaborée dans une matière mal connue, ou bien une porcelaine d'origine orientale ou bien encore une variété d'agate: Petronius Arbiter ad libidinis concitamentum myrrhinum se poculum bibisse refert; « Pétrone Arbiter rapporte qu'il but dans une coupe myrrhine afin d'éveiller son désir ». Or le débauché Malchion qui est la cible de la satire 82 du livre 3 fait exactement de même et sert son vin vieux dans des coupes myrrhines: Opimianum morionibus nectar / Crystallinisque murrinisque propinat (Martial 3, 82, 24-25). Ce vin «opimien » est daté par le consulat 
d'Opimius en 21 avant J.-C., une année proverbialement exceptionnelle, mais évidemment bien trop lointaine pour avoir été réellement bue encore à l'époque de Martial, ainsi que le confirme Velleius Paterculus qui, sous Tibère, faisait déjà la même constatation $^{16}$.

Ce que l'on ne dit pas, à ma connaissance, c'est que c'est précisément le même vin que se fait servir Trimalchion au cours de la cena: Statim allatae sunt amphorae uitreae diligenter gypsatae, quarum in ceruicibus pittacia erant affixa cum hoc titulo: Falernum Opimianum annorum centum (sat. 34, 6): "Sur le champ on apporta des amphores en verre bouchées avec soin aux cols desquels avaient été accrochées des étiquettes avec cette inscription : "Falerne Opimien - Cent ans d'âge". "

Il me paraît donc certain qu'il faut abandonner l'identification du Petronius Arbiter donné comme auteur du Satyricon par les manuscrits avec le Petronius tacitéen des chapitres 17-19 du livre 16 des Annales. Jamais en effet Tacite ne présente ce haut personnage comme un homme de lettres et il n'aurait assurément pas manqué de le faire si la chose avait eu quelque réalité. La formule si commentée de ann. 16, 18, elegantiae arbiter, ne prouve rien. Ou plutôt si : elle explique tout. Le passage de Tacite en question a très probablement inspiré l'auteur du Satyricon qui a écrit son roman sous un pseudonyme. On sait combien la tradition littéraire occidentale est riche de pseudonymes cryptés, ironiques ou humoristiques, et je citerai, pour me cantonner à la période contemporaine, simplement le nom d'Émile Ajar ou celui d'Edgar Faure : le premier, connu aussi sous le nom de Romain Gary, non seulement remporta deux prix Goncourt sous ses deux hypostases pour Les Racines du ciel en 1956 et pour La Vie devant soi en 1975, mais encore choisit deux noms qui ont en commun leur étymologie russe et leur signification associée au feu pour le premier, à la braise pour le second. On sait en revanche peu que l'ancien ministre de la $\mathrm{IV}^{\mathrm{e}}$ et de la $\mathrm{v}^{\mathrm{e}}$ République Edgar Faure fut un excellent auteur de romans policiers: Pour rencontrer M. Marsches et L'installation $d u$ président Fritz-Mole. Le plus connu reste M. Langlois n'est pas toujours égal à lui-même qu'il publie sous le pseudonyme d'Edgar Sanday (Sandé) parce que son prénom s'écrivait sans la lettre D.

La chronologie et l'histoire de la réception des lettres de Pline le Jeune dans l'Antiquité doit probablement être revue. On estime en effet généralement que le Panégyrique de Trajan, par son éclat, a éclipsé la correspondance. Personne n'aurait ainsi lu les lettres de Pline entre les $\mathrm{II}^{\mathrm{e}}$ et $\mathrm{IV}^{\mathrm{e}}$ siècle ${ }^{17}$, soit avant Symmaque à la fin du $\mathrm{IV}^{\mathrm{e}}$ siècle et la connaissance par l'orateur païen du corpus plinien paraît assurée ${ }^{18}$. Comme l'écrit Philippe Bruggisser, «Symmaque aurait été sensible à l'analogie entre les souffrances que les sénateurs ont endurées sous le joug de Domitien et sous celui de Valentinien I $^{\text {er }}$ et les espoirs qu'on fait naître le règne de Nerva et celui de Gratien ${ }^{19}$ ». Il est vrai que Pline le Jeune lui-même, s'il raconte les succès des lectures qu'il donnait de ses discours (epist. 3,$18 ; 4,5)$ ou de ses poèmes (epist. 8, 21) n'évoque jamais la moindre recitatio de ses lettres. L'histoire de la publication des Lettres ne peut être faite, même s'il est hautement vraisemblable que Pline y a pourvu lui-même, sans doute à une date tardive.

Ce que je voudrais à présent avancer c'est que les lettres de Pline eurent très tôt un lecteur attentif, son contemporain, l'auteur du Satyricon. 
9 Le premier Luigi Pepe avait, en 1958, suggéré avec force que l'auteur du Satyricon avait lu la correspondance de Pline et il appuyait son hypothèse sur la ressemblance entre sat. 115, 6 et la lettre de Pline à Tacite décrivant l'éruption du Vésuve (epist. 6, 20, 19) ${ }^{20}$. Mais son article ne semble pas avoir eu grand retentissement. René Martin à son tour relève certains liens thématiques qui existent entre l'œuvre de Pline le Jeune et le Satyricon: le motif par exemple de la captatio des héritages (epist. 8,18$)$ ou encore celui des loups-garous ou autres striges et fantômes (epist. 7, 27) ${ }^{21}$. Il faut les enregistrer avec attention.

10 Mais surtout l'identification par René Martin de l'auteur du Satyricon en la personne d'un affranchi de Pline le Jeune, un intellectuel érudit du nom d'Encolpius, me paraît lumineuse dans son évidence et absolument probante ${ }^{22}$. J'ajoute à présent, dans les pages qui suivent, quelques nouveaux arguments en faveur de cette thèse.

11 Cet homme, Encolpius, membre de la familia de Pline au sens premier du mot latin, faisait aussi partie de ses « familiers » au sens courant du terme en français moderne : il comptait au nombre des intimes de son maitre. On voit ce dernier se promener dans ses propriétés entouré des membres de sa familia, parmi lesquels de vrais intellectuels : mox cum meis ambulo, quorum in numero sunt eruditi ${ }^{23}$. Les fonctions de lector exercées par Encolpius (epist. 8,1,2) doivent être comprises dans la plus grande extension possible du terme. On lisait en effet dans la société de ce temps à haute voix ${ }^{24}$. Titinius Capito, le contemporain et correspondant de Pline, lisait lui-même ses ouvrages ${ }^{25}$. Mais Pline pour sa part lisait mal, surtout les vers, et on lui en faisait le reproche : explica aestum meum; audio me male legere, dumtaxat uersus ${ }^{26}$. Aussi faisait-il appel à l'un de ses affranchis, peut-être Encolpius, pour faire connaître ses vers à ses amis : cogito ergo recitaturus familiaribus amicis experiri libertum meum. Hoc quoque familiare quod elegi non bene, sed me melius, scio, lecturum ${ }^{27}$ : «Je songe par conséquent pour une lecture que je compte faire à des amis proches à tester mon affranchi. Ce que j'ai choisi lui est aussi familier et il le lira, je le sais, non pas parfaitement mais mieux que moi. " Encolpius faisait en outre à son maître les lectures qui agrémentaient les divers moments de ses journées et il s'agit cette fois des ouvrages d'autrui : cenanti mihi, si cum uxore uel paucis, liber legitur ${ }^{28}$. Les auteurs lus par Encolpius doivent être ceux que mentionne Pline dans ses lettres et l'on retrouve ainsi l'explication des parallèles enregistrés entre le Satyricon et Quintilien, Martial ou Silius Italicus ${ }^{29}$.

Cet homme - ou ses pareils s'ils étaient plusieurs - était ainsi placé par ses fonctions au cœur de l'activité intellectuelle de Pline et savait tout non seulement des productions de son maître, qu'elles soient en cours de gestation ou achevées, mais encore de l'actualité littéraire par le biais des lettres reçues par Pline ou des ouvrages qu'on adressait en hommage à l'une des personnalités les plus en vue de son temps ${ }^{30}$. On connaît cette anecdote piquante rapportée par Pline et qui prouve que Tacite et luimême passaient pour les deux plus grands écrivains de leur temps ${ }^{31}$. Comment Encolpius aurait-il pu ignorer dans ces conditions la proximité de Pline avec Tacite ? Il avait transcrit, lu, corrigé peut-être les onze lettres de Pline à Tacite ${ }^{32}$ et il avait lu les pages des Annales envoyées par Tacite à son ami comme il était informé de la parution des Histoires (epist. 9, 27, 1) 33 . Cela ne fait aucun doute : c'est là qu'il a trouvé l'idée d'un Arbiter Petronius, fabriqué par jeu littéraire en associant Petronius et elegantiae arbiter (ann. 16, 18).

13 L'auteur du Satyricon a choisi son nom de "plume » par référence à un passage bien caractérisé de l'ouvrage historique du plus grand historien vivant de son temps : Tacite. 
Comme Trimalchion qui affirme dans son épitaphe funéraire n'avoir jamais entendu la leçon d'aucun philosophe ${ }^{34}$, Petronius, chez Tacite, meurt «sans vouloir écouter ni réflexions sur l'immortalité de l'âme ni propos de philosophes ${ }^{35}$ " mais plutôt des "poésies légères et des vers gais ${ }^{36}$ ». Loin de prouver que Petronius est l'auteur du Satyricon, je considère plutôt que ces propos ont plu par leur étrangeté et leur ton inhabituel à l'amateur de plaisanteries qui y a trouvé le prétexte du pseudonyme adopté pour signer son roman.

Peut-être peut-on avancer une ultime explication: le consulaire Petronius fut, aux dires de Tacite gouverneur de la province de... Bithynie ${ }^{37}$, la région même à la tête de laquelle Pline le Jeune a été nommé par Trajan en 111 ! Formons une hypothèse: Encolpius, l'affranchi de Pline, libéré de la présence de son patron qui, absent pendant deux ou trois ans, décède en Bithynie en 113 ou 114, a relâché sa surveillance sur sa domus, a bien pu mettre à profit cette demi-liberté, loin de la censura tutélaire du maître, pour composer le Satyricon qu'il faudrait dès lors placer après 111. Dans ce cas de figure Pline n'aurait jamais rien su de l'existence d'un roman bien trop scandaleux pour avoir été présenté à son approbation.

L'auteur du Satyricon est donc un affranchi et non un aristocrate. Ainsi s'effondrent définitivement toutes les hypothèses de lecture fondées sur la prétendue supériorité de caste de l'auteur et son mépris pour le monde vulgaire, grossier et inculte des liberti dont Trimalchion serait l'emblème majeur et à peine caricaturé. Il convient désormais de conserver toujours à l'esprit qu'Encolpius (auteur du Satyricon) appartient au monde même de Trimalchion, ce monde avide de reconnaissance sociale et d'affirmation de soi par l'argent si bien décrit par Paul Veyne ${ }^{38}$. Ainsi s'explique qu'Encolpe (personnage du Satyricon) n'accepte pas que les critiques de ses deux compères Ascylte et Giton à l'encontre de Trimalchion aillent trop loin dans la cruauté : il juge leurs quolibets déplacés (sat. 57, 1 : intemperantis licentiae) et indécents (58,1: indecenter) et les rappelle à l'ordre au nom d'une solidarité de classe qui ne dit pas son nom.

C'est dans la correspondance de Pline que l'on trouve le meilleur témoignage pour tenter de comprendre ce qu'un intellectuel devait ressentir à subir la morgue d'un aristocrate qui faisait perpétuellement peser sur lui le joug de la dépendance financière. Il ne s'agit de rien d'autre que de cette lettre appelée parfois à tort «Éloge de Martial » dans laquelle Pline pose en défenseur des Lettres et des Arts. On annonce à Pline le décès de l'épigrammatiste. L'épistolier se livre alors à un éloge bref qui le conduit très vite à l'objectif réel et unique de la lettre : rappeler les vers dans lesquels Martial a fait l'éloge de son talent littéraire, raison pour laquelle cette epistula $21 \mathrm{du}$ livre 3 devrait en réalité s'appeler «Éloge de Pline par Martial» et non "Éloge de Martial par Pline »! Ces dix vers, en outre, Pline les cite complaisamment in extenso (epist. 3, 21, 4), ce qui se comprend bien puisque Martial ne fait pas moins que de comparer, pour leur qualité, les discours de Pline à ceux du grand Cicéron ${ }^{39}$. Mais ce n'est pas tout : Pline rappelle encore au passage qu'il a aidé financièrement Martial au moment du départ du poète pour sa patrie : prosecutus eram uiatico secedentem; « je lui avais fait un don, au moment de son départ, pour son voyage » et qu'il s'est ainsi conformé à la vieille et noble tradition du mécénat des patroni en faveur de leurs clients. Et puis in cauda uenenum: la lettre s'achève en effet par une pique à la fois brillante et méprisante. Après avoir fait mine de croire l'espace d'un instant que les œuvres de Martial passeraient à la postérité, Pline dénonce férocement les illusions du poète : - At non erunt aeterna quae scripsit? Non erunt fortasse, ille tamen scripsit tamquam 
essent futura ; « Mais, diras-tu, ses œuvres ne seront pas éternelles ? Sans doute que non, mais lui, il les a écrites dans l'idée qu'elles le seraient ! ».

Pour nous l'ironie de Pline fait le charme de ses lettres, comme elle le fait de la Correspondance de Madame de Sévigné lorsque la malignité s'empare d'elle, ce qui arrivait quelquefois, ou de certaines lettres de Gide à Claudel, qu'il détestait. Mais que devait penser d'une telle attitude notre Encolpius qui ne pouvait sans doute s'interdire de rapprocher de son propre cas le mépris exprimé par Pline envers un eruditus de seconde classe? À l'appui de l'identification d'Encolpius avec l'auteur du Satyricon citons ce passage en vers du début du roman dans lequel le vieux professeur d'Encolpe fait une analyse de la décadence de l'enseignement pleine de bon sens et donne à l'apprenti écrivain de sages conseils, parmi lesquels celui-ci :

Nec curet alto regiam trucem uultu

cliensue cenas inpotentium captet,

nec perditis addictus obruat uino

mentis calorem; neue plausor in scenam

sedeat redemptus histrioniae addictus ${ }^{40}$.

"Qu'il ne se préoccupe pas des demeures hautaines où l'on porte le menton haut; qu'il n'aille point en client fréquenter les festins de tyrans ni se livrer aux débauchés et perdre sous l'effet de la boisson la force de son esprit ; qu'il n'aille pas, payé pour cela, faire la claque et applaudir assis devant la scène la comédie que l'on donne.»

Je crois que ces vers, sincères de la part non seulement d'Agamemnon mais aussi d'Encolpius, expriment le grand désenchantement voire l'amertume d'un intellectuel contraint par sa situation sociale et ses origines serviles de continuer à servir après son affranchissement même. Que dénoncent ces vers? La situation même d'Encolpius, l'auteur du Satyricon. Il n'est question ici ni de théâtre véritable ni d'histrion au sens propre. La scène évoquée est celle du monde de Pline et l'histrion est le maître auquel son lector est condamné jour après jour à servir flagorneries et autres petitesses. Il ne saurait y avoir d'intellectuel que libre et la métaphore du vin des festins - la vie dans l'entourage de Pline n'avait rien d'une bacchanale - n'est qu'un travestissement. L'essentiel est dit : un écrivain, un homme de lettres, un intellectuel ne saurait vendre l'indépendance de son génie (mentis calor) à aucun prix. Agamemnon dénonce la prostitution littéraire du temps et Encolpius la sienne propre. On comprend mieux désormais pourquoi il le fait sous un pseudonyme!

19 Je crois profondément que l'ambiguïté sociale du statut d'affranchi qui est celui de son auteur explique un grand nombre des aspects jugés jusqu'ici peu compréhensibles du Satyricon. La situation sociale et la position intellectuelle d'Encolpius, à la fois proche de Pline et l'admirant, fasciné en tout cas par ce que représente le personnage dans le monde littéraire du temps, mais essentiellement méprisé de lui, le sachant parfaitement, en souffrant sans doute, cette ambiguïté de classe donc qui est à la source des sentiments tiraillés $\mathrm{du}$ lector pour son patron expliquent l'ambiguïté consubstantielle du Satyricon et son ironie souvent indiscernable, toujours indécidable dans les passages en vers par exemple.

Après tout la clef est dans le roman. Trimalchion affirme avoir vécu pendant quarante années comme esclave sans que personne sache s'il était réellement un esclave ou un homme libre : annis quadraginta seruiui; nemo tamen scit utrum seruus essem an liber (sat. 57, 9). On tire de ce témoignage l'idée que les affranchis étaient bien traités, à l'égal d'hommes libres. J'en retiens tout autre chose : la difficulté d'être de ces hommes privés 
de toute individualité et identité propres. Ce n'est pas un habit facile à endosser que celui d'ancien quelque chose.

Le lector de Pline est ainsi ou bien un esclave particulièrement apprécié, choyé, distingué, promis à un affranchissement à une date lointaine ou bien un affranchi demeuré là où son histoire personnelle l'avait enraciné sans qu'il l'ait choisi. Sa personnalité réelle s'est révélée ailleurs que dans son rôle de lector. De conseiller culturel de son maitre il en est devenu le mémorialiste clandestin. L'Histoire offre de nombreux autres exemples de secrétaires devenus les historiens de leur maître, depuis Las Cases dans le Mémorial de Sainte-Hélène jusqu'à Claude Mauriac témoin, dans le Temps

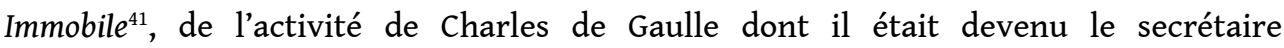
particulier, à Paris, entre 1945 et $1948^{42}$. Encolpius raconte sa vie dans la maison de Pline, celle d'amis ou de connaissances. Voilà en partie le sujet initial du Satyricon, devenu sous l'effet de la puissance créatrice de leur auteur des mémoires fantasmés.

J'en veux pour preuve les nombreuses convergences thématiques entre la correspondance de Pline et le roman d'Encolpius. J'ai commencé tout à l'heure par la crise que connaît de son temps l'enseignement des lettres aux dires d'Encolpe. Disons pour mémoire que Pline le Jeune pensait exactement la même chose et dénonçait cette décadence en mettant en valeur le rôle des quelques intellectuels qui résistaient, parmi lesquels son ami Titinius Capito, procurateur ab epistulis de Trajan (CIL 6, 798), un peu pompeusement désigné comme le rédempteur d'une situation désespérée: ipsarum denique litterarum iam senescentium reductor ac reformator (epist. 8, 12, 1); « il est celui qui sauve les lettres aujourd'hui en déclin et les revivifie ».

On écrit ensuite des épopées dans le Satyricon. Qu'elles soient miniatures, sous forme de ce que la tradition littéraire appelait des epyllia, comme par exemple les vers en hexamètres dactyliques par lesquels Agamemnon, après avoir emprunté à la tradition métrique de la satire (sat. 5 , vers 1-8: des choliambes), chante cette fois sur le mode héroïque le programme d'enseignement qu'il voudrait voir suivre par tous les élèves de rhétorique (sat. 5, vers 9-22), faisant au passage même l'éloge sans ambiguïté et sans aucune ironie du genre épique (vers 19 : Dent epulas et bella truci memorata canore; "Que les guerres offrent l'occasion de festins littéraires, elles qui sont dignes d'être chantées sur un ton guerrier ») à la composition duquel il voudrait voir les hommes de lettres se consacrer. Ou qu'elles soient de longue haleine comme les 295 vers du poème d'Eumolpe sur les guerres civiles (sat. 119-124). Or dans l'entourage de Pline on écrivait l'épopée. Son correspondant Caninius s'apprêtait à rédiger un ouvrage sur la guerre de Trajan contre les Daces ${ }^{43}$. Il ne fait aucun doute qu'il devait s'agir d'une épopée ${ }^{44}$ et qu'elle devait être en vers grecs (epist. 8, 4, 3 : graecis uersibus) imités d'Homère dont le patronage est invoqué dans ce même passage ${ }^{45}$. En outre, et c'est une conjonction remarquable avec la thématique épique dans le Satyricon, Pline lui-même a écrit des vers héroïques : expertus sum me aliquando et heroo (epist. 7, 4,3); « je me suis jadis essayé au vers héroïque ». Et puis il y a ses fameux hendécasyllabes, auxquels Pline tient tant, dont il raconte la genèse (epist. 7, 4, 3-4) et desquels il prend grand plaisir à citer un échantillon substantiel (epist. 7, 4, 6). Pourquoi n'a-t-on jamais assez insisté sur le fait que le Satyricon lui-même ne comptait pas moins de neuf passages dans ce mètre ${ }^{46}$ et que le nombre total des hendécasyllabes dont le roman est truffé se monte à 59 vers? 
Ajouterai-je encore à cette liste déjà nourrie ? On lit en sat. 71, 1-12 le testament rédigé par Trimalchion et en sat. 141, 2 celui d'un autre affranchi anonyme (Eumolpe ?). Or Pline autorisait ses affranchis à faire des testaments, ce qui est une libéralité notable par rapport au droit en vigueur au $\mathrm{II}^{\mathrm{e}}$ siècle ${ }^{47}$ : permitto seruis quoque quasi testamenta facere eaque ut legitima custodio (epist. 8, 16, 1) ; « j'autorise mes esclaves aussi à faire des espèces de testaments et je les respecte comme s'ils étaient légaux ».

Voici encore, par exemple, un parallèle de situation qui n'a, à ma connaissance, jamais été relevé entre les deux documents. Au début de la Cena, alors qu'Encolpe et ses compagnons sont sur le point de pénétrer dans le triclinium de leur hôte, cet espace si difficile d'accès ${ }^{48}$, ce saint des saints protégé par divers gardiens à la manière des Champs Élysées d'un enfer sur terre ${ }^{49}$, ils sont arrêtés une nouvelle fois par la survenue inopinée d'un esclave se jetant à leurs pieds. Ce malheureux implore leur pitié et leur demande d'intercéder en sa faveur. Il a en effet commis une négligence, se laissant dérober les vêtements du trésorier qui avaient été confiés à sa garde. Encolpe et ses compagnons vont trouver le dispensator, obtiennent son pardon et se voient remerciés par l'esclave gracié. Que signifie cet épisode peu éclairé par les travaux de la critique? J'y vois pour ma part avant tout un reflet à peine déguisé de pratiques sociales du monde de Pline le Jeune. Il suffit en effet de parcourir la correspondance de ce dernier pour y reconnaître tous les motifs de la saynète du Satyricon. Simplement ce qui se passe entre hommes libres chez Pline se passe entre esclaves chez Pétrone. De même que dans la comédie plautinienne le monde des serui singe celui des ingenui, ou encore l'univers des valets celui des aristocrates comme dans le chef-d'œuvre de Renoir, $L a$ Règle du jeu. Pline aimait afficher sa sollicitude pour les difficultés ou les maladies de ses esclaves et c'est précisément à propos d'une indisposition d'Encolpius qu'il se déclare à l'écoute : nos solliciti (epist. 8, 1,3); « nous sommes plein d'attention ». On connaît aussi cette lettre fameuse dans laquelle Pline prétend traiter à table ses affranchis comme des convives parmi les autres, c'est-à-dire des hommes libres, leur servant notamment le même vin qu'à tous ${ }^{50}$. Le propos de Trimalchion en sat. 71,1 n'est-il pas un écho de cette protestation d'humanité : et serui homines sunt? Sauf que chez Pétrone - trait d'humour en forme de clin d'œil - ils boivent non le même vin que les hommes libres mais le même... lait (sat. 71, 1 : aeque unum lactem biberunt)!

Le thème de l'écoute attentive prêté aux affranchis considérés comme des êtres d'une égale dignité est donc éminemment plinien : il est même mis en scène par l'épistolier qui affirme sa sollicitude dans un dialogue savamment orchestré avec un interlocuteur imaginaire ${ }^{51}$ ad maiorem gloriae sui. Le lexique même de la page du Satyricon qui nous retient est celui de Pline. Car qu'illustre la scène du roman ? L'importance du pardon au sein d'une société d'affranchis, ce que leurs maîtres nomment indulgentia ou clementia. Pour ne pas alourdir mon dossier de références que l'on accroîtrait à loisir, par exemple en puisant dans le Panégyrique de Trajan, je me contenterai de citer l'entame de l'epistula 19 du livre 5 de Pline: Video quam molliter tuos habeas, quo simplicius tibi confitebor qua indulgentia meos tractem; « je vois avec quelle douceur tu agis avec tes affranchis; aussi vais-je te faire l'aveu en toute simplicité de l'indulgence avec laquelle j'agis envers les miens ». Ce motif, on le constate, était ainsi l'objet d'une véritable surenchère entre aristocrates. On ne saurait non plus négliger le témoignage de l'epistula 14 du livre 8 qui montre Pline plaidant, contre les partisans de la peine de mort à l'origine majoritaires, en faveur de l'absolution pour les affranchis du consul Afranius Dexter accusés sans 
preuve du meurtre de leur maître ${ }^{52}$. Il est incontestable que telle fut la position de l'orateur au Sénat et qu'il fit triompher au terme de la bataille sa position ${ }^{53}$.

La différence notable avec la scène du Satyricon est la nature du délit : d'une accusation de meurtre (c'est aussi le cas dans l'affaire Larcius Macedo dans laquelle Pline prend encore une position modéré $\left.{ }^{54}\right)$ on est passé à un larcin véniel. Les habits dérobés au trésorier ne valent pas un liard ${ }^{55}$. La preuve, c'est qu'ils avaient déjà subi une fois l'épreuve du lavage qui déprécie tant les vêtements ${ }^{56}$. Le Satyricon a beau être réaliste (c'est-à-dire qu'il est inspiré par la réalité du monde plinien), il est aussi parodique (il transpose cette réalité sur un mode comique). La grandeur de Pline et sa générosité emplie de dignité pour ses affranchis est ici tournée en dérision par le moyen de l'écart entre la grandiloquence des gestes (on se jette comme dans la tragédie aux pieds des grands que l'in implore: procubuit ad pedes, sat. 30, 7) ainsi que l'emphase des discours des personnages (peccatum suum propter quod periclitaretur) et la petitesse du larcin. Ce type d'écart est un ressort bien connu de toutes les formes de comique.

Mais la parodie est encore plus fine et Pline semble bien visé. C'est ce que tendent à faire penser les concordances lexicales. L'esclave gracié couvre ses bienfaiteurs de ses remerciements et loue leur bonté : gratias agens humanitati nostrae (sat. 31, 2). On pourrait songer qu'ici Encolpius prononce un discret plaidoyer pro domo, à savoir qu'il affirme l'humanité pleine et entière des affranchis qui ne sont pas des sous-hommes. Peut-être. Mais surtout on retrouve là le thème plinien de l'humanitas d'un maître modèle et empreint de bonté pour les siens. Lorsque Pline sait son affranchi Zosime souffrant c'est son humanitas qu'il veut lui témoigner: Quod si essem natura asperior et durior, frangeret me tamen infirmitas liberti mei Zosimi, cui tanto maior humanitas exhibenda est quanto nunc illa magis est (epist. $5,19,2)$; «Même si ma nature était plus sévère et rude, je serais néanmoins touché par la maladie de mon affranchi Zosime à qui je dois d'autant plus manifester ma compassion qu'elle lui est en ce cas précis davantage utile ». Et même attitude de Pline, même lexique encore dans une lettre où l'épistolier s'apitoie sur les maladies de ses affranchis (epist. 8, 16, 1: confecerunt me infirmitates meorum; «les maladies des miens m'ont affligé »). Il s'affirme vaincu et brisé par la compassion (humanitas) qu'il ne peut s'empêcher d'éprouver : debilitor et frangor eadem illa humanitate quae me ut hoc ipsum permitterem induxit (epist. $8,16,3) ;$ « je suis détruit et brisé par cette compassion-là qui m'a conduit à m'autoriser cette faiblesse même ».

Le lexique de Pétrone dans l'épisode du vol des hardes du trésorier n'a aucune cohérence avec le caractère véniel de l'incident. Ce lexique est en réalité celui de Pline, ce même lexique qui traverse les panégyriques: poenas eripere; periclitare; deprecati sumus; remittere poenam; obligati tam grandi beneficio; gratias agens humanitati nostrae... On croirait lire le De clementia de Sénèque ou encore un De officiis quelconque, peut-être même le Panégyrique de Trajan. Ce lexique et ces valeurs sociales de reconnaissance liés au pardon d'une faute sont ceux d'un traité de morale, ce que les lettres de Pline, à leur manière, cherchent précisément à être.

Le trésorier dont on implore la clementia est peint avec des traits empruntés à la littérature édifiante la plus sérieuse et ses airs superbes appartiennent aux Grands qu'ils singe : superbus ille sustulit uultum (sat. 30,10). Sa réponse pourrait passer pour celle d'un prince ou d'un patronus sinon autocrate du moins gonflé de sa responsabilité et de sa dignitas: non tam iactura me mouet quam neglegentia nequissimi uiri. C'est ici du grand théâtre, digne de celui que Pline mettait en scène dans ses lettres. Le motif de la iactura de grand prix si elle n'est pas réparable apparaît en effet en epist. 6, 8, 6 où il est 
question d'une dette que Atilius Crescens cherche à recouvrer : grauis est ei uel minima iactura quia reparare quod amiserit grauius est; " la moindre perte lui est lourde étant donné qu'il a de la peine à retrouver ce qu'il a perdu ». Là encore la parodie dans le roman pétronien réside dans l'application du motif à un objet facile à remplacer, des nippes de vil prix. Ailleurs c'est ainsi au décès d'un ami que s'applique iactura chez Pline : iacturam grauissimam feci si iactura dicenda est tanti uiri amissio (epist. 1, 12, 1) ; «j'ai subi une grande perte si le mot perte n'est pas trop faible pour évoquer la disparition d'un si grand homme».

Et puis, pour emporter définitivement la conviction - mais est-ce encore nécessaire ? citons ce billet de Pline qui raconte comment un affranchi de la maisonnée de son ami Sabinianus est venu le trouver pour implorer son pardon: Libertus tuus, cui suscensere te dixeras, uenit ad me aduolutusque pedibus meis tamquam tuis haesit. Fleuit multum, multum rogauit, multum etiam tacuit, in summa fecit mihi fidem paenitentiae (epist. 9, 21); "Ton affranchi, contre lequel, disais-tu, tu t'es mis en colère, est venu me trouver et s'est jeté à mes pieds comme il l'aurait fait aux vôtres et demeura prostré. Il pleura abondamment, supplia abondamment, se tut aussi longtemps et, à la fin, m'a convaincu qu'il se repentait ». Même situation : un affranchi vient supplier l'indulgence d'un tiers et se jette à ses pieds; même lexique (sat. 30, 7 : procubuit ad pedes ac rogare coepit ; Pline, epist. 9, 21 : aduolutus pedibus meis... multum rogauit).

On a déjà, pour finir, remarqué l'attention prêtée à la fois par l'auteur du Satyricon et par Pline le Jeune aux questions liées aux testaments. Il en va de même pour un aspect un peu particulier des testaments, le choix de son vivant des conditions d'inhumation dans un tombeau. Pline raconte ainsi sa tristesse à constater que le tombeau de Verginius Rufus, plus de dix ans après sa disparition en $97^{57}$, demeure inachevé. Ce qui navre Pline est que Verginius avait rédigé lui-même son inscription funéraire, laquelle rappelait qu'il avait refusé d'usurper l'empire au moment de la révolte de Vindex en 68 et qu'il cite en sa mémoire. La conclusion de l'épistolier consiste à donner le conseil à son destinataire Albinus de songer à se prémunir de son vivant de l'incurie de ses héritiers et de pourvoir lui-même à tous les soins de la construction d'un tombeau : ut ispi nobis debeamus etiam conditoria exstruere omniaque heredum officia praesumere (epist. 6, $10,5)$; « aussi devons-nous nous-mêmes édifier nos sépulcres et remplir par avance les devoirs de nos héritiers ». Or Trimalchion - ou Encolpius, l'auteur du roman - a entendu la leçon de Pline: non seulement il prend soin de faire connaître avant sa disparition l'épitaphe qu'il a lui-même composée à sa mémoire $(s a t .71,12)$, mais encore il prévoit dans le détail les modalités de construction de son futur tombeau, de la dimension du monument aux éléments d'ornement (sat. 71, 6-7). Par souci de dérision comique le tombeau de Trimalchion sera aussi vaste (cent pieds par deux cents ${ }^{58}$ ) que celui de Verginius Rufus était modeste ${ }^{59}$.

En outre Trimalchion donne la consigne à Habinnas de s'occuper de cela dès à présent. Il le fait, dit-il, dans le but « d'éviter de subir, une fois décédé, l'infamie » (sat. 71, 8 : ne mortuus iniuriam accipiam) que constituerait un oubli de leur devoir de la part de ses héritiers, à l'instar de ce qui arriva à Verginius Rufus. Par quel mot Pline avait-il qualifié le préjudice subi par Verginius ? Celui-là même dont use Trimalchion : iniuria (epist. 6, 10,6). Le rapprochement n'est pas dû au hasard mais constitue un indice supplémentaire et sûr des relations d'influence entre les lettres de Pline et le Satyricon. Et puis Trimalchion en héritant à la mort de son maitre " un patrimoine de sénateur " $($ sat. 76,2$)$ héritait aussi de son gentilice. Ainsi «quand il a légué sa fortune à son 
affranchi favori, le patron de Trimalchion a pu se dire qu'après tout celui-ci était le dernier qui perpétuât son nom et que les biens ainsi légués ne sortiraient pas du nom ${ }^{60}$ ». Paul Veyne fait ici allusion à la formule enregistrée par le Digeste $(32,94)$ par laquelle un patron lègue à ses affranchis des biens-fonds « à condition qu'ils ne sortent pas du nom ", ne de nomine suo exirent. Or Pline pose exactement la même limite au droit d'hériter qu'il accorde à ses esclaves : ils peuvent « faire des dons et des legs » dumtaxat intra domum (epist. 8, 16, 2), «à condition que rien ne sorte de la maison ». La destinée de Trimalchion telle qu'il la raconte lui-même est donc parfaitement conforme aux pratiques recommandées par Pline.

Comment s'en étonner si Encolpius, le lector de Pline, est bien, comme l'a brillamment démontré René Martin et comme je crois l'avoir confirmé par de nouveaux rapprochements inédits et irréfutables entre la correspondance de Pline et le roman, l'auteur du Satyricon?

Dérision et parodie chez Pétrone, l'analyse n'est pas nouvelle. Ce qui l'est, c'est l'identification précise des éléments parodiés et de la cible du roman : le monde plinien. L'univers de Pline ou le microcosme de sa familia est une image en réduction de la société aristocratique romaine, de ses valeurs et de ses comportements ${ }^{61}$. Or le monde des affranchis de Pline est lui-même, aux dires de l'épistolier en personne, un état ou une ciuitas en réduction : nam seruis res publica quaedam et quasi ciuitas domus est (epist. 8, $16,2)$; « car pour mes esclaves ma maison est pour ainsi dire un État et une espèce de cité ». On ne saurait mieux dire. Placé au cœur de cette cité familière Encolpius, l'affranchi de Pline, son secrétaire chargé des affaires littéraires, l'auteur du Satyricon, en est l'observateur privilégié et le peintre amusé.

La domus de Pline le Jeune est ainsi la ciuitas de Pétrone.

\section{NOTES}

1. V. HUGO, Les Misérables, éd. HETZEL-QUANTIN, Paris, s. d. (1881), vol. 3, p. 141. L'éditeur de Victor Hugo, J. HETZEL, ajoute ailleurs, dans sa Préface aux Châtiments que ses poèmes «comme les Annales de Tacite, comme les Satires de Juvénal, sont un livre d'éducation pour les peuples, - ces enfants qui ont tant de peine à mûrir ", Les Châtiments, seule édition complète, Hetzel, Paris, s. d. (1869), p. III.

2. A. ERNOUT, Le Satiricon, Paris, CUF, 1923.

3. A. ERNOUT, p. VII.

4. A. ERNOUT, note 1, p. 2.

5. A. ERNOUT, note 2, p. 3.

6. A. ERNOUT, note 1, p. 4.

7. Pensées 23, p. 1094 CHeVAlier (Pensées 3 BRUnSCHVICG), Paris, 1954 (Bibliothèque de la Pléiade). 
8. Cf. notamment "Qui a (peut-être) écrit le Satyricon?", Revue des Études Latines 78, 2000, p. 139-163 ; et ID., Le Satyricon. Pétrone, Paris, 1999.

9. Pour Martial, cf. déjà A. CoLligon, Étude sur Pétrone. La critique littéraire, l'imitation et la parodie dans le Satiricon, Paris, 1892, p. 391-395.

10. Martial 3, 82, 32 : Hoc Malchionis patimur inprobi fastus.

11. Frg. 18, p. 185-186 ERNOUT.

12. Cf. aussi Juvénal 8, 85-86: dignus morte perit, cenet licet ostrea centum / Gaurana et Cosmi toto mergatur aeno.

13. R. MARTIN, "Qui a (peut-être) écrit le Satyricon?" (cité note 8), p. 148.

14. P. 183 ERNOUT.

15. Sat. 16-18. C'est une glose qui figure dans le mss Parisinus 7975 qui donne une double précision précieuse : les vases murrhins sont fournis par Quartilla et cet épisode prenait place au livre 14 du roman : cf. apparat critique du frg. 8, p. 183 ERNOUT.

16. Velleius Paterculus 2, 7, 56 : Hic est Opimius, a quo consule celeberrimum Opimiani uini nomen; quod iam nullum esse spatio annorum colligi potest, cum ab eo sint ad te, M. Vinici, consulem anni CLI. Factum Opimii, quod inimicitiarum quaesita est ultio, minor secuta auctoritas, et uisa ultio priuato odio magis quam publicae uindictae data.

17. Panorama complet de "La postérité des Lettres de Pline le Jeune" par É. wolfF, Pline le Jeune ou le refus du pessimisme, Rennes, 2003, p. 95-98.

18. Cf. Ph. BRUGGISSER, Symmaque ou le rituel épistolaire de l'amitié littéraire. Recherches sur le premier livre de la correspondance, Fribourg, 1993, p. 110-111 (idéal de la simplicitas) et p. 224-225.

19. Symmaque (cité note 16), p. 225.

20. L. PEPE, "Petronio conosce l'epistolario di Plinio", Giornale Italiano di Filologia 11, 1958, p. 289-294.

21. R. MARTIN, “Qui a (peut-être) écrit le Satyricon?" (cité note 8), p. 150.

22. R. MARTIN, "Qui a (peut-être) écrit le Satyricon?" (cité note 8), p. 156-159; cf. Pline, epist. 8, 1, 1-3. Afin d'éviter toute ambiguïté je nomme, dans la suite de cette étude, «Encolpius » l'auteur du Satyricon et « Encolpe » le personnage du roman.

23. Epist. 9, 4.

24. Epist. 8, 13 : Probo quod libellos meos cum patre legisti.

25. Epist. 8, 12 : recitaturus est Titinius Capito quem audire nescio magis debeam an cupiam.

26. Epist. 9, 34, 1.

27. Epist. 9, 34, 1.

28. Epist. 9, 36, 4.

29. Quintillien est cité par Pline dans epist. 2, 14, 9 ; Martial dans epist. 3, 21 ; Silius Italicus dans epist. 3, 7.

30. Ouvrage envoyé à Pline, cf. par exemple epist. 9, 1, 2;9, 22, 1-2;9, 28, 3 ;9, 31 ; composition envoyée par Pline, cf. par exemple epist. 9, 4, $1 ; 9,18,2 ; 9,20,1 ; 9,25$. Sur les insertions des compositions de Pline dans les ouvrages de ses amis et inversement, cf. par exemple epist. 9, 11, 1. - Sur Pline écrivain on pourra consulter le très suggestif ouvrage d'É. wolfF, Pline le Jeune (cité note 17), p. 83-98.

31. Epist. 9, 23 : Tacite a raconté à Pline, qui, fort flatté, le rapporte à son tour, qu'un chevalier assis à ses côtés aux jeux du cirque l'a confondu avec Pline.

32. Epist. 1,$6 ; 1,20 ; 4,13 ; 6,9 ; 6,16 ; 6,20 ; 7,20 ; 7,33 ; 8,7 ; 9,10 ; 9,14$.

33. Il est tout à fait possible que les fonctions de lector ne fussent pas très éloignées des charges d'un notarius ou d'un scribe : cf. epist. 9, $36 ; 9,40$.

34. Sat. 71,12 : nec umquam philosophum audiuit.

35. Ann. 16, 19, 2 : audiebatque referentes nihil de immortalitate animae et sapientium placitis.

36. Ann. 16, 19, 2 : sed leuia carmina et faciles uersus. 
37. Ann. 16, 19, 2 : proconsul tamen Bithyniae.

38. "Vie de Trimalcion", La société romaine, Paris, 1991, p. 13-87.

39. Epist. 3, 21, 4 :... posterique possint / Arpinis quoque comparare chartis.

40. Sat. 5, 4-8.

41. Volume 5, Aimer De Gaulle, Paris, 1978.

42. Cf. C. MAURIAC, Conversations avec André Gide. Extraits d'un Journal, Paris, 1951, p. 263-264, Lettre à André Gide du 4 janvier 1945 : «Car il se trouva ceci : que mon ami Claude Guy sur la vie duquel je m'étais chaque jour anxieusement interrogé au cours de ces quatre années, était devenu l'aide de camp du général, et qu'il fit appel à moi, le premier soir pour l'aider, car si vite avaient été les événements que de Gaulle arrivait à Paris presque seul. C'est ainsi que je participai d'aussi près que possible aux heures historiques qui suivirent. Et que, n'ayant accepté ce poste, pour lequel je ne me trouvais point qualifié, que provisoirement et pour dépanner mon ami, je me trouve encore au Cabinet du général à ce jour, et chef de son secrétariat particulier. »

43. Epist. 8, 4, 1 : optime facis quod bellum Dacicum scribere paras.

44. Epist. 8, 4, 3: Vna sed maxima difficultas quod haec aequare dicendo arduum, immensum etiam tuo ingenio, quamquam altissime adsurgat et amplissimis operibus increscat.

45. Epist. 8, 4, 3 : si datur Homero et mollia uocabula.

46. Sat. 15, $9: 2$ vers ; 79, $8: 5$ vers ; 93, $2: 10$ vers ; 109, $10: 7$ vers ; frg. $25: 4$ vers ; frg. 28,9 vers ; frg. $54: 10$ vers; frg. $55: 5$ vers; frg. $61: 7$ vers.

47. Cf. J. GAUDEMET, Institutions de l'Antiquité, Paris, 1967, p. 563-564. La loi Papia Poppaea en 9 après J.-C. avait augmenté les garanties du patron en lui donnant le droit de conserver une partie de l'héritage d'un affranchi : cf. Gaius 3, 41-42.

48. Sat. 30, 5 : cum conaremur in triclinium intrare ; le verbe d'effort marque la difficulté à parvenir jusque-là.

49. Premier obstacle: le procurator aux comptes (sat. 30, 1); deuxième obstacle: l'esclave «superstitieux» (sat. 30, 5); troisième obstacle: l'esclave suppliant (sat. 30, 7). Le chien représenté sur le mur (sat. 29, 1 : in pariete pictus) est bien sûr un autre Cerbère.

50. Epist. 2, 6, 3-4.

51. Epist. 2, 6, 3-4:- Eadem omnibus pono; ad cenan enim, non ad notam inuito cunctisque rebus exaequo, quos mensa et toro aequaui. - Etiamne libertos? - Etiam; conuictores enim tunc, non libertos puto. - Et ille: Magno tibi constat. - Minime. - Quid fieri potest ? - Potest quia scilicet liberti mei non idem quod ego bibunt, sed idem ego quod et liberti.

52. Epist. 8, 14, 12 .

53. Epist. 8, 14, 23 : obtinui quidem quod postulabam.

54. Epist. 3, 14. Dans le récit de cet épisode tragique du meurtre d'un maître par ses esclaves Pline fait d'une part une allusion à la cruauté de Larcius Macedo envers ses esclaves et à son désir de revanche sociale sur l'humiliation subie jadis par son père, un ancien esclave lui-même $(3,14,1$ : superbus alioqui dominus et saevuus et qui seruisse patrem suum parum, immo nimium meminisset) et rappelle d'autre part la nécessité de l'humanitas ou de la «douceur» envers ses esclaves, condition nécessaire sinon suffisante $(3,14,5:$ nec est quod quisquam possit esse securus quia sit remissus et mitis ; non enim iudicio domini sed scelere perimuntur).

55. C'est le sens de uix dans sat. 30, 8 : quae uix fuissent decem sestertiorum.

56. Sat. 30, 11 : iam semel lota.

57. Epist. 6, 10 : on peut donc dater cette lettre après 107.

58. Sat. 71, $6:$ praeterea ut sint in fronte pedes centum, in agrum pedes ducenti.

59. Epist. 6, 10, 3 : opus modicum ac exiguum.

60. VEYNE, "Vie de Trimalcion" (cité note 38), p. 23.

61. VEYNE, "Vie de Trimalcion" (cité note 38), p. 61-62. 


\section{RÉSUMÉS}

L'auteur du Satyricon connaissait bien la correspondance de Pline le Jeune. Il y a puisé un certain nombre de thèmes ou de situations caractéristiques de la vie intellectuelle du début du second siècle ainsi que de la condition des affranchis. Ces parallèles fournissent un argument sûr pour refuser l'identification de l'auteur du Satyricon avec le consulaire Petronius mentionné par Tacite. Ils établissent que le roman a été écrit après l'année 107 et peut-être après 111, très probablement au début du second siècle. Ils confirment enfin de manière éclatante la thèse qui voit en Encolpius, l'affranchi de Pline le Jeune, l'auteur du Satyricon.

The author of the Satyricon did actually know Pliny the Younger's correspondence. He drew from it a certain number of themes or characteristics of the intellectual life, also of the condition of the freed slaves at the beginning of the second century. Those parallels provide a safe argument against identifying the author of the Satyricon with the consular Petronius mentioned by Tacitus. They establish that the novel was written after the year 107 and perhaps after 111, very likely at the beginning of the second century. Lastly they strikingly confirm the assumption that sees in Encolpius, Pliny the Younger's freed man, the author of the Satyricon.

INDEX

Mots-clés : Pétrone, Satyricon, date de rédaction et auteur, Encolpius, Pline le Jeune

Keywords : Petronius, Satyricon, date of writing and author, Encolpius, Pliny the Younger

\section{AUTEUR}

\section{STÉPHANE RATTI}

Professeur à l'université de Bourgogne

stephane.ratti@u-bourgogne.fr 